\title{
Cavernous Hemangioma of the Nasal Cavity
}

\author{
Peter Kalina*, Jeffrey Rykken \\ Department of Radiology, Mayo Clinic, Rochester, USA \\ E-mail: *kalina.peter@mayo.edu \\ Received November 1, 2011; revised November 30, 2011; accepted December 8, 2011
}

\begin{abstract}
A 22 year old six month pregnant female presented with right eye tearing, proptosis and nasal congestion. CT revealed a large right nasal cavity mass with involvement of the ethmoids, right maxillary sinus, lamina papyracea, cribriform plate and nasal septum. There was significant remodeling of the right cribriform plate with mild extra-axial intracranial and mild intraorbital extension. Transnasal endoscopic excision confirming the diagnosis of cavernous hemangioma of the nasal cavity. Hemangiomas are benign slow-growing vascular neoplasms classified as capillary, cavernous or mixed. Hemangiomas of the nasal cavity and paranasal sinuses are very rare with only a few reported cases. The occurrence and growth of these lesions during pregnancy may be related to increased blood volume or hormonal factors. The most common therapeutic option is complete surgical resection via transnasal endoscopic approach. Pre-operative embolization may be utilized in some cases to decrease the risk of intraoperative bleeding.
\end{abstract}

Keywords: Cavernous Hemangioma, Nasal Cavity

\section{Case Report}

A 22 year old female presented with several months of progressive right eye tearing, proptosis and nasal congestion. She was six months pregnant. Computed Tomography (Figures 1 and 2) revealed a $4.5 \times 4 \times 3.5 \mathrm{~cm}$ well circumscribed mass originating in the right nasal cavity, involving the ethmoids with remodeling of the medial wall of the right maxillary sinus, lamina papyracea, cribriform plate and nasal septum. The turbinates on the right were not visualized due to replacement by the mass. There was significant remodeling of the right cribriform

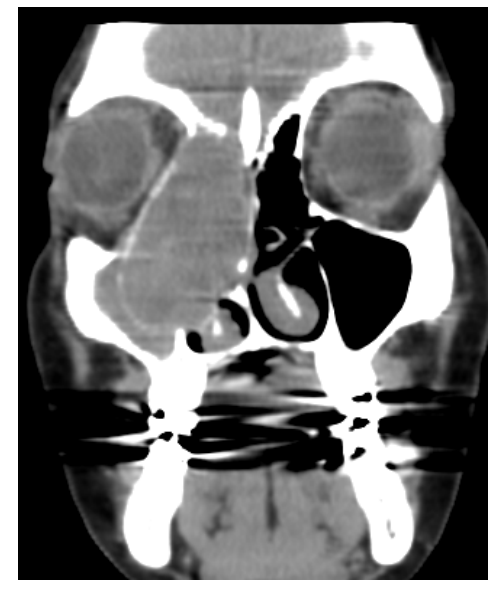

(a)

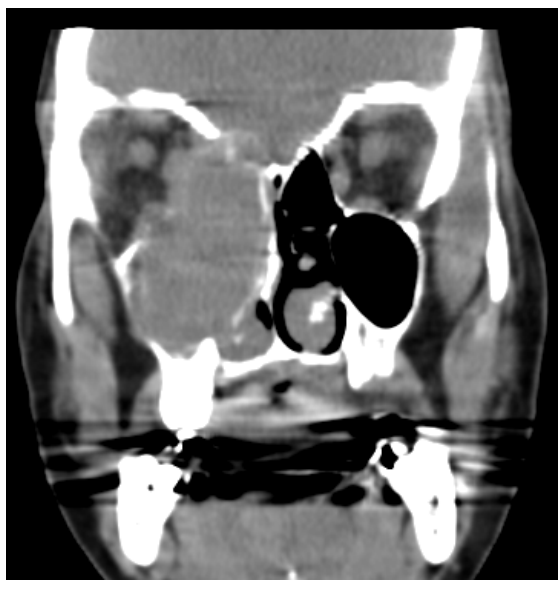

(b)

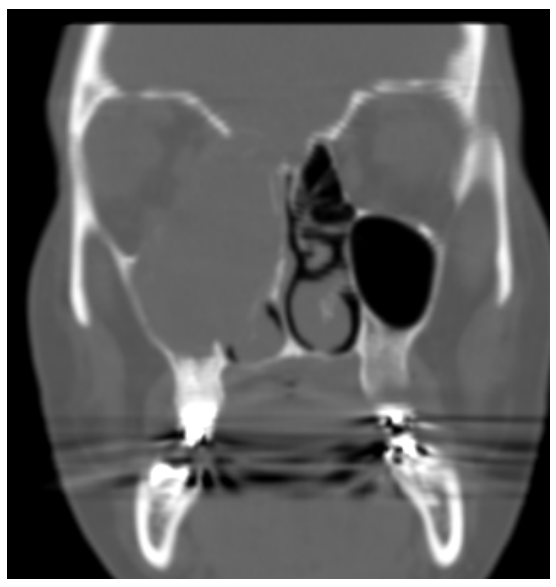

(c)

Figure 1. 1(a) and 1(b) (soft tissue windows) and 1(c) (bone windows) in the coronal plane as well as Figure 2(a) (soft tissue) and 2(b) (bone in the axial plane demonstrate a $4.5 \times 4 \times 3.5 \mathrm{~cm}$ well circumscribed mass centered in the right nasal cavity, involving the ethmoids with remodeling of the medial wall of the right maxillary sinus, lamina papyracea, cribriform plate and nasal septum). There is significant remodeling of the right cribriform plate (1(c)) with mild extra-axial intracranial extension (1(b)). The lesion abutted the right periorbita with mild intraorbital extension (1(b)). 


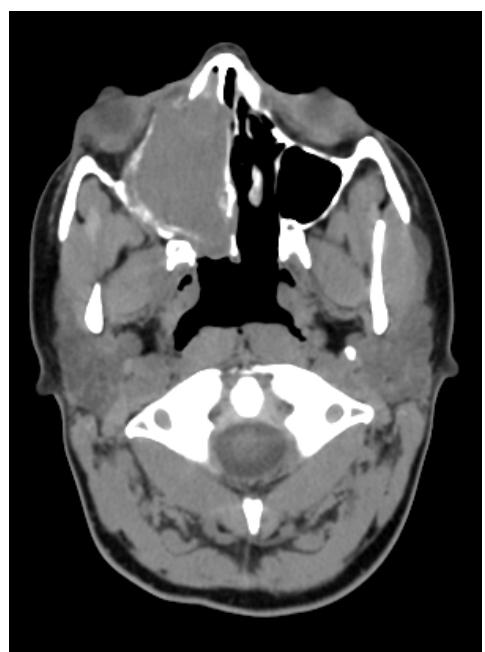

(a)

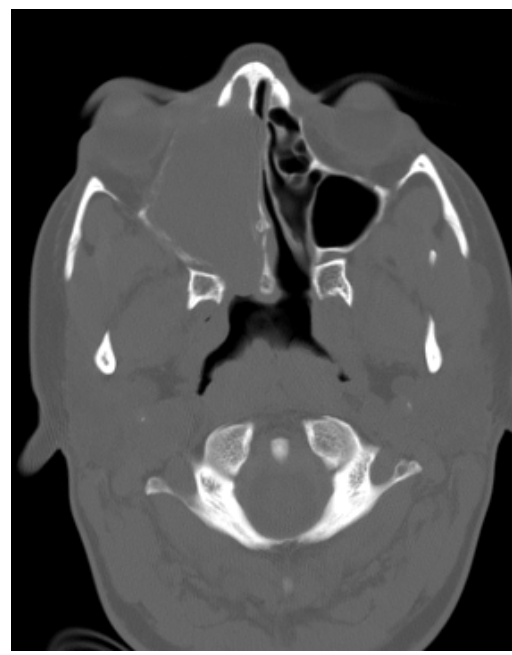

(b)

Figure 2. (a) (soft tissue) and (b) (bone in the axial plane demonstrate a $4.5 \times 4 \times 3.5 \mathrm{~cm}$ well circumscribed mass centered in the right nasal cavity, involving the ethmoids with remodeling of the medial wall of the right maxillary sinus, lamina papyracea, cribriform plate and nasal septum).

plate with mild extra-axial intracranial extension. The lesion abutted the right periorbita with mild intraorbital extension. The right frontal sinus and right maxillary sinus were obstructed with fluid and membrane thickening. Transnasal endoscopic biopsy was utilized in order to arrive at a definitive diagnosis and subsequent complete excision. Histologic examination confirmed the surgical findings of cavernous hemangioma of the nasal cavity.

\section{Discussion}

Hemangiomas are benign slow-growing vascular neoplasms. They are classified as either capillary, cavernous or mixed. Although hemangiomas are commonly found in the head and neck, hemangiomas of the nasal cavity and paranasal sinuses are relatively uncommon with only a few case reports in the literature [1-4]. Both cavernous and capillary types of hemangiomas have been reported to occur in the sinonasal region. This is only the second such case reported to originate from the mucosa rather than being primarily intraosseous [2]. This is the first such case to demonstrate intracranial and intraorbital extension. Although, the exact relationship is not entirely certain, the occurrence and growth of these lesions during pregnancy may be related to increased blood volume. Alternatively, hormonal factors may be contributing factors. Differential diagnostic considerations for the CT appearance of a nasal cavity hemangioma would include nasal polyp, antrochoanal polyp, inverted papilloma, hemangiopericytoma, lymphoma and esthesioneuroblastoma. Therapeutic options for these lesions vary although the most common is that of complete surgical resection via transnasal endoscopic approach $[3,4]$. Pre-operative embolization may be utilized in some cases to decrease the risk of intraoperative bleeding. Because the clinical and imaging findings are nonspecific, surgery may be necessary for not only treatment but also to obtain a definitive diagnosis.

\section{References}

[1] F. Caylakli, A. Cagici, C. Hurcan, et al., "Cavernous Hemangioma of the Middle Turbinate,” Ear Nose Throat Journal, Vol. 87, No. 7, 2008, pp. 391-393.

[2] M. Archontaki, A. Stamou, M. Kalomenopoulou and D. Korkolis, "Cavernous Hemangioma of the Left Nasal Cavity,” Acta Otorhinolaryngologica Italica, Vol. 28, No. 6, 2008, pp. 309-311.

[3] K. Takeda, Y. Takenaka and M. Hashimoto, "Intraosseous Hemangioma of the Inferior Turbinate," Case Reports in Medicine, Vol. 2010, 2010, Article ID 409429. doi:10.1155/2010/409429

[4] K. Akiyama, M. Karaki, Y. Osaki, et al., "Intraossseous Cavernous Hemangioma of the Middle Turbinate,” Auris Nasus Larynx, Vol. 38, No. 4, 2011, pp. 516-518. doi:10.1016/j.anl.2010.10.010 